\title{
Solving the Problem of Thermodynamic Inequalities
}

\author{
Authors: V.A Etkin ${ }^{1 *}$ \\ Etkin Valeriy Abramovich, Togliatti State University, Scientific Center, Advisor to the Vice-Rector for Science \\ E-mail: 1etkin.v@mail.ru
}

\begin{abstract}
It is shown that the combined equation of the 1 st and 2 nd principles of classical thermodynamics does not transform into inequality in the case of irreversible processes, if the external energy exchange of the system is expressed in terms of energy carrier flows. This means that thermodynamic inequalities are generated by attempts to take into account the irreversibility of real (non-static) processes, without taking into account explicitly its reasons - the inhomogeneity of the system and the presence of internal sources not only for entropy, but also for other parameters.

On this basis, exact expressions of heat and work in open nonequilibrium systems, as well as their dissipative function, are obtained. The physical meaning of entropy as a thermal impulse and the unprovability of the principle of its increase in the framework of equilibrium systems are revealed. Non-entropy criteria for evolution are proposed and the latter is shown to be incompatible not only with the second law of thermodynamics, but also with the laws of conservation of energy carriers. The elimination of thermodynamic inequalities opens up the possibility of applying the equations of thermodynamics, taking into account energy dissipation, to other fundamental disciplines.
\end{abstract}

Keywords: Thermodynamics; inequalities; irreversibility; the principle of increasing entropy; evolution criteria; conservation laws.

\section{Introduction}

To date, enough evidence has accumulated in natural science that testifies to the fact that nature is characterized not only by a destructive, but also by a creative tendency. However, both classical thermodynamics [1,2] and thermodynamics of irreversible processes (TIP) $[3,4]$ still claim that biological and technical systems tend to "chaos", and the Universe - to "thermal death". "The question of the physical foundations of the law of monotonous increase in entropy" ... remains open [5]

In modern natural science, even a new concept has arisen that claims the existence of two diametrically opposite physical principles that control processes in objects of animate and inanimate nature. If you follow it, science will have to abandon the notion of the existence of universal laws of nature, valid for any material systems.

The beginning of the difficulties that arose, in our opinion, was the division by the founder of classical thermodynamics R. Clausius of all forms of energy exchange of the system with the environment for heat $\mathrm{Q}$ and work $\mathrm{W}$, which led to the formulation of the law of conservation of energy in the form [6]:

$$
d \bar{U}=\delta Q-\delta W
$$

where $\delta Q, \delta W$ are elementary amounts of heat $\mathrm{Q}$, brought to an equilibrium system with energy $\bar{U}$, and the work $W$ done by it.

At the same time, R. Clausius distinguished heat $Q$ as a quantitative measure of the heat transfer process (for brevity, "process heat") and heat $Q^{\partial}$ released in the system due to the performance of "disgregation" $W^{\partial}$ (for brevity, "body heat"). He called their sum "total heat", however, after a series of discussions, they began to call it "internal energy" $U$. This required finding the specific coordinate of the heat transfer process $\delta Q$, that is, a parameter that necessarily changes during heat exchange and does not change otherwise kind of R. Clausius found such a parameter, considering the cycle of a reversible (ideal) heat engine, and calling it the entropy $S$. However, he found that if the efficiency of a real heat engine (including a heat source, its receiver and working fluid) is considered smaller than that of reversible, then the entropy of such a system at the end of the cycle will increase. Thus, if in reversible processes $\delta Q=T d S$, then in irreversible processes $\delta \mathrm{Q}>T d S$. In this case, equation (1) should be written as:

$$
T d S d \bar{U}+\delta W,
$$

where the "=" sign refers to reversible processes, and the ">" sign refers to irreversible processes.

With an increase in the intensity of processes, inequalities of type (2) intensify, and the 
calculation of the heat $Q$ and the work $W$ based on the $1 \mathrm{st}$ and $2 \mathrm{nd}$ principles of classical thermodynamics becomes more and more inaccurate. Moreover, this theory itself is not able to estimate the error associated with neglect of the indicated inequalities, since their exact analytical expressions remain unknown. This problem is compounded by the transition to open systems and complex (multivariate) systems, which, in addition to the expansion work, perform other types of work $W_{k}$ (for example, the work of introducing $k$ substances and charge into the system, the work against surface tension forces, the work of polarization, magnetization, etc.). In this case, the masses of the components of the $k$-th substance $M_{k}$ (due to internal chemical transformations), the coordinates $\Theta_{k}$ of these works (due to relaxation of the system), and also their momenta (due to the action of external forces) can change spontaneously. This excludes the possibility of calculating the external energy exchange of the system (heat transfer, mass transfer, and work) using classical methods, which forces fundamental disciplines to confine themselves to studying reversible processes.

In this regard, it is of undeniable interest to show that the occurrence of thermodynamic inequalities was the result of errors of a methodological nature and to propose methods for describing and measuring external energy exchange by parameters of not the state of the system, but this process itself. Below we propose a solution to this problem from the standpoint of thermokinetics, a theory that generalized TIP to locally nonequilibrium systems and processes of useful energy conversion [7].

\section{The basic equation of thermodynamics of the nonequilibrium continuum}

We will proceed from the energy conservation law $U$ of the "extended system", which includes the object of study and its environment, in the form proposed by N. Umov (1873) [8]:

$d U / d t+\oint \boldsymbol{j}_{u} \cdot d \mathbf{f}=0$

where $U$ is the internal) energy of the object of study (system) of constant volume $V$ extracted from the environment by a closed surface $\mathbf{f} ; \boldsymbol{j}_{u}$ (W $\mathrm{m}^{-2}$ ) is the density of its flow through the vector element df of its surface in the direction of the external normal $\boldsymbol{n}$.

According to the concept of short-range inherent in this equation, the energy $U$ does not just disappear at some points in space and arises at others, but is transferred by some energy carriers
$\Theta_{k}$ ( $k$-th substances in the number of $N_{k}$ moles, their masses $M_{k}$, entropy $S_{k}$, charges $\Theta_{e}$, momenta $\boldsymbol{P}_{k}$, etc.) through the boundaries of the system. This form of the law of conservation of energy takes into account the kinetics of real processes, without making any assumptions about the mechanism of energy transfer and the internal structure of the system, and therefore is the most general.

We now take into account that the $\boldsymbol{j}_{u}$ stream is composed of the $j$-th energy flows $\boldsymbol{j}_{u k}$ of the $k$-th kind $U_{k}$, each of which is in turn expressed by the product of the energy carrier $\boldsymbol{j}_{k}$ and its potential $\psi_{k}$ $=d U_{k} / d M$ (specific energy), i.e., $\boldsymbol{j}_{u k}=\psi_{k} \boldsymbol{j}_{k}=$ $\psi_{k} \rho_{k} \boldsymbol{v}_{k}$, where and $\boldsymbol{v}_{k}$ is the transfer rate of the $k$-th energy carrier $\Theta_{k}$ through the fixed boundaries of the system, $\boldsymbol{j}_{u}=\sum_{k \boldsymbol{j}_{u k}}=\sum_{k} \psi_{k} \boldsymbol{j}_{k}, \quad(k=1,2, \ldots K)$ is its density. Then

$\boldsymbol{j}_{u}=\Sigma_{k} \boldsymbol{j}_{u k}=\Sigma_{k} \psi_{k} \boldsymbol{j}_{k},(k=1,2, \ldots K)$.

Using the Gauss-Ostrogradsky theorem, we transform expression (1) to the form $d U / d t+$ $\int \nabla \cdot \dot{j}_{u} d V=0$. After decomposing $\nabla\left(\psi_{k} \dot{j}_{k}\right)$ into independent components $\Sigma_{k} \psi_{k} \nabla \cdot \dot{j}_{k}+\Sigma_{k} \boldsymbol{j}_{k} \cdot \nabla \psi_{k}$, the energy conservation law (1) appears in the form: $d U / d t+\Sigma_{k} \int \psi_{k} \nabla \cdot \mathbf{j}_{k} d V+\Sigma_{k} \int \mathbf{j}_{k} \cdot \nabla \psi_{k} d V=0$,

If the mean value of the potential $\psi_{k}$ and the average value $\boldsymbol{X}_{k}$ of the local thermodynamic force $\boldsymbol{x}_{k}=-\nabla \psi_{k}$, expressed by the negative gradient of the potential $\psi_{k}$ are taken out of the integral sign, then equation (3) can be represented in terms of the parameters of the system as a whole, as is customary in classical thermodynamics [2]:

$d U / d t+\Sigma_{k} \Psi_{k} J_{k}-\Sigma_{k} \boldsymbol{X}_{k} \cdot \boldsymbol{J}_{k}=0$. (BT)

Here $J_{k}=\oint \boldsymbol{j}_{k} d \mathbf{f}=\int \nabla \cdot \boldsymbol{j}_{k} d V$ is the scalar flow of the $k$-th energy carrier from the system, corresponding to the concept of its "flow"); $\boldsymbol{J}_{k}=$ $\int \rho_{k} \boldsymbol{v}_{k} d V=\Theta_{k} \overline{\boldsymbol{v}}_{k}$ is the vector flow of the same energy carrier, corresponding to the concept of its momentum.

Expression (6) divides the energy exchange of the system with the environment into two fundamentally different parts. The first sum characterizes the quantitative change in the energy of the $k$-th energy carrier $U_{k}=\int_{\psi_{k} \rho_{k}} d V$, caused by the addition of the energy carrier $\Theta_{k}$, the second a qualitative change in this energy, due to a change in its potential $\psi_{k}$. These two categories of energy transfer processes are referred to in thermokinetics as energy transfer and energy conversion [7].

In equilibrium systems $\left(\boldsymbol{X}_{k}, \boldsymbol{x}_{k}=0 ; \Psi_{k}=\psi_{k}\right) J_{k}$ $=\oint \boldsymbol{j}_{k} \cdot d \mathbf{f}=-d \Theta_{k} / d t$, and expression (6) goes over into the combined equation of the 1 st and 2 nd laws 
of the classical thermodynamics of multivariate systems in the form of the generalized Gibbs relation [4]:

$d U=\Sigma_{k} \psi_{k} d \Theta_{k}$.

A particular case of this expression is the classical Gibbs relation for open multicomponent systems [2]:

$d \bar{U}=T d S-p d V+\Sigma_{k} \mu_{k} d N_{k}$,

where $T, p, \mu_{k}$ is the absolute temperature, pressure, and chemical potential of the $k$-th component of the equilibrium system.

As we see, the law of conservation of energy of nonequilibrium systems in the form of (6) does not turn into inequality, despite the explicit inclusion in it of the non-static (irreversibility) of the processes under consideration. This solves the most important "problem of thermodynamic inequalities," which so far has hindered the application of thermodynamics to real (occurring at a finite speed) processes and other fundamental disciplines. By finding the fluxes $\boldsymbol{J}_{k}$ and the forces $\boldsymbol{X}_{k}$ on a more general basis of the energy conservation law (6), it is possible to extend the concepts and methods for taking into account the irreversibility of TIP to systems that carry out targeted energy conversion [7].

It is also important that this equation returns to thermodynamics the concept of force, which has become redundant in it due to the quasi-static nature of the processes under study. At the same time, it gives the forces $\boldsymbol{X}_{k}, \boldsymbol{x}_{k}$ and the generalized process rates (flows $\boldsymbol{J}_{k}, \boldsymbol{j}_{k}$ ) a single and unambiguous meaning of the average and local field strengths of the corresponding energy carrier $\Theta_{k}$ and its momentum. In this case, any of these pulses can be decomposed into a translational, rotational, and oscillatory component. Thus, the total number of degrees of freedom of a multivariate system is $3 K$ and can reach arbitrarily large, although finite. Along with the exclusion of any arbitrariness in the choice of $\boldsymbol{J}_{k}$ flows and $\boldsymbol{X}_{k}$ forces, this allows us to create a single system of physical quantities for all fundamental disciplines, which opens up the prospect of a true revolution in metrology. However, here we restrict ourselves to only those of the consequences of eliminating inequalities that affect the foundations of thermodynamics itself.

\section{Finding accurate expressions of heat and work in open systems.}

The solution to the problem of thermodynamic inequalities involves, first of all, finding analytical expressions for heat transfer, mass transfer, and work under conditions of the simultaneous occurrence of these processes in the system. We begin by defining the concept of the heat of the process $Q$ under open nonequilibrium systems. In classical thermodynamics, it is often defined as "that which is not work," that is, "by the reverse balance." This is also the case in the thermodynamics of irreversible processes (TIP), where the heat flux $\boldsymbol{J}_{q}$ and its density $\boldsymbol{j}_{q}$ are found by subtracting from the flux of the internal energy flux associated with the diffusion of $k$-substances through the boundaries of the system (i. e, with selective mass transfer) [3,4]. This definition corresponds to the understanding of heat in open systems as that part of the energy exchange that is not related to the transfer of matter across the boundaries of the system [1]. However, there is serious disagreement in finding this part. More often than others, the value [9] is taken as the heat flux vector:

$\boldsymbol{j}_{q}=T\left(\boldsymbol{j}_{s}-\Sigma_{k} \mu_{k} \dot{j}_{k}\right)$,

where $\boldsymbol{j}_{s}, \boldsymbol{j}_{k}$ is the flux density of the entropy and $k$ th substance.

However, since convective energy transfer in open systems depends on the conditions of the process), some authors propose to subtract the term $\Sigma_{k} S_{k} N_{k}$, from the total change in the entropy of the system $d S$, others the term $\Sigma_{k} h_{k} N_{k}$, and others the term $\Sigma_{k} u_{k} N_{k}$ (where $\mathrm{s}_{k}, h_{k}$ and $u_{k}$ are respectively the partial molar entropy, enthalpy and internal energy of the $k$-th substance [9]. Naturally, this leads to an ambiguous calculation of heat transfer.

The situation is not the best with the work of expansion in open systems since a change in the density $\rho_{k}$ in them can occur in the case of a constant volume. This requires the coordinates of independent processes to be unchanged while other independent processes occur. As applied to open systems, this requirement means that the coordinates of heat transfer and work in (1) should be represented through parameters that remain unchanged under conditions of input of $k$ substances. The total entropy $S$ and the volume of the system $V$ are not such quantities since they also change during mass transfer. The specific entropy $\mathrm{s}$ and the volume of the mixture $v$ are not them since they also change with the diffusion of $k$ substances (i.e., with a change in the composition of the system). Finally, the partial molar entropies and the volume of the kth components $\mathrm{s}_{k}$ and $v_{k}$ cannot serve as coordinates of heat transfer and 
work in open systems, since they also change with a change in the composition of the system.

To overcome the difficulties that have arisen, it is necessary to subtract from the full increment $S$ and $V$ not only that part of $\Sigma_{k} s_{k o} d N_{k}$ and $\Sigma_{k} v_{k o} d N_{k}$, which is due to the transfer of pure $k$-th substances with specific entropy $\underline{s}_{k o}$ and volume $v_{k o}$ across the boundary but also that part $\left(s_{k}-s_{k o}\right)$ and $\left(v_{k}-v_{k o}\right)$, which is caused by thermal and volume effects arising from the diffusion of interacting components in the system itself, that is, by their internal sources [10]. This part is expressed by the difference between the actual increment of entropy and the volume of the mixture when a mole of the kth substance is introduced (characterized by the partial molar entropy $s_{k}$ and the partial molar volume $v_{k}$ ) and the corresponding values of $\underline{s_{k o}}$ and $v_{k o}$ for the pure substances of which the mixture is composed. This approach corresponds to the choice of the reversibly composed mixture (the components of which do not interact or are separated from each other by a movable heat-conducting partition) as the coordinate of heat transfer and the work entropy $\underline{s}_{k o}$ and volume $v_{k o}$. Since these parameters are functions of temperature and pressure only, this corresponds to the classical definition of heat and work in closed multicomponent systems in the absence of mixing processes.

The result of this definition is the following expressions for the heat flux $\boldsymbol{j}_{q}$ and the volumetric strain momentum $\boldsymbol{j}_{v}[10]$ :

$\boldsymbol{j}_{q}=T \Sigma_{k} \rho_{s o} \boldsymbol{v}_{s o}=T \boldsymbol{j}_{s o} ; \quad \boldsymbol{j}_{v}=\Sigma_{k} \rho_{k o} \boldsymbol{v}_{k o}=p \nabla \cdot \boldsymbol{v}_{o}$,

where $T, p$ are local values of the absolute temperature and pressure in a given section of the system boundary; $\rho_{k o}, \rho_{s o}$ is the density of the $k$-th component and its entropy.

\section{The failure of the hypothesis of local equilibrium}

The absence of the concept of the force $\boldsymbol{X}_{k}$ and time $t$ as a physical parameter in classical thermodynamics made it difficult to find criteria for the conditions of equilibrium and stationarity in it, forcing it to resort to the conditions of extremality of entropy and several other thermodynamic potentials. This drawback was overcome by the creation of the theory of irreversible processes (TIP) in the twentieth century, where the generalized rates of various relaxation processes in adiabatically isolated systems (flows $J_{i}$ ) were expressed as the time derivatives of some parameters $\mathrm{A}_{i}$, which characterize the distance of an adiabatically isolated system from equilibrium, and the generalized forces $X_{i}$ were expressed as derivatives of the flows $S$ from the entropy of the system [11]. However, serious difficulties arose due to the absence of such parameters $A_{i}$ in thermodynamics and other fundamental disciplines. Progress in this direction was achieved thanks to the introduction by I. Prigogine of the hypothesis of local equilibrium [12]. This hypothesis assumed the presence of equilibrium in the elements of spatially heterogeneous systems (despite the occurrence of macro processes in them), the possibility of describing their state with the same set of variables as in equilibrium (despite the appearance of scalar $X_{i}$ and vector $\boldsymbol{X}_{i}$ "thermodynamic" forces) and validity for these elements of all equations of equilibrium thermodynamics (despite their inevitable transition to inequalities in the case of non-static processes). Despite such a deep internal inconsistency, this hypothesis made it possible to find expressions for the sought-after thermodynamic forces and flows and based on them to justify all other provisions of the TIP. True, for this I had to resort to drawing up, based on other fundamental disciplines, cumbersome equations of the balance of energy, mass, charge, and momentum, from which we could then compose the equation of balance of entropy and find the "production" of entropy $d_{i} S / d t$ and by not arbitrarily breaking it into factors $X_{i}$ and $J_{i}$ find the desired variables of the nonequilibrium state [4].

Meanwhile, this most time-consuming procedure could be avoided by knowingly recognizing the need to introduce additional parameters to specify the state of an inhomogeneous continuum. Indeed, in the general case of nonequilibrium systems, the coordinates of the independent processes $\Theta_{k}$ can change both as a result of the transfer of the corresponding energy carrier through the boundaries of the system $d_{e} \Theta_{k} / d t=-J_{k}=-\int \nabla \cdot \dot{j}_{k} d V$, and due to the presence of their source inside the system $d_{i} \Theta_{k} / d t=\int \sigma_{k} d V$ with density $\sigma_{k}$ :

$$
d \Theta_{k} / d t=d_{e} \Theta_{k} / d t+d_{i} \Theta_{k} / d t=-\int \nabla \cdot \mathbf{j}_{k} d V+\int \sigma_{k} d V
$$

In particular, the number of moles of $k$-th chemical elements or their compounds $N_{k}$ change during chemical reactions, momenta $\boldsymbol{P}_{k}=M_{k} \boldsymbol{v}_{k}$ of any $k$-substances - under the influence of longrange forces, volume $V$ - when expanding into a void without doing work, etc. If we substitute the balance equations (11) in (6), we obtain:

$d U / d t=\Sigma_{k} \Psi_{k} d \Theta_{k} / d t-\Sigma_{k} \int \psi_{k} \sigma_{k} d V+\Sigma_{k} \boldsymbol{X}_{k} \cdot \boldsymbol{J}_{k}$. (Вт) (12) 
This directly implies the relationship between the sources and sinks of various energy carriers $\Theta_{k}$ under stationary irreversible processes $(d U / d t=0$; $\left.d \Theta_{k} / d t=0\right)$ :

$\Sigma_{k} \int \psi_{k} \sigma_{k} d V=\Sigma_{k} \int \boldsymbol{x}_{k} \cdot \dot{j}_{k} d V$; или $\Sigma_{k} \Psi_{k} d_{i} \Theta_{k} / d t=$ $\Sigma_{k} \boldsymbol{X}_{k} \cdot \boldsymbol{J}_{k}$.

According to this equation, the interconversion of energy is caused precisely by a change in energy carriers and therefore affects not only the entropy of the system but also other quantitative measures of motion in the system. In this case, the presence of a source or sink of a specific energy carrier $\Theta_{k}$ depends on the sign of the product $\boldsymbol{X}_{k} \cdot \boldsymbol{J}_{k}$, which is positive for relaxation processes $\left(\boldsymbol{X}_{k} \cdot \boldsymbol{J}_{k}>0\right)$, and negative for work performed "against equilibrium" in the system $\left(\boldsymbol{X}_{k} \cdot \boldsymbol{J}_{k}<0\right)$. This reveals the limitations of TIP, which takes into account only the relaxation component of real processes.

\section{The inadmissibility of the transformation of entropy into a "scapegoat" for any all irreversibility.}

A special case of expression (13) under conditions when all other energy carriers $\Theta_{k}$ obey the conservation laws $\left(\sigma_{k}=0\right.$ for $\left.k \neq s\right)$ is the entropy balance equation

$d S / d t=d_{e} S / d t+d_{i} S / d t=-\int \nabla \cdot \mathbf{j}_{s} d V+\int \sigma_{s} d V$

where $d_{e} S, d_{i} S$ are the changes in the entropy $d S$, caused respectively by the heat exchange of the system and its "production" due to irreversibility [9]. Moreover, (13) takes the form of a dissipative function

$T d_{i} S / d t=\Sigma_{k} \boldsymbol{X}_{k} \cdot \boldsymbol{J}_{k}>0$.

This halo of "exclusivity" still attracts both specialists and amateurs to entropy. Meanwhile, it can be shown that entropy is simply an impulse of the oscillatory motion of particles of a substance $P$ $=M v$, which has lost its vector nature due to the randomness of thermal motion [7]. This momentum exists for any of the energy carriers $\Theta_{k}$ in any of their aggregate states, turning into an impulse of ordered translational and rotational motion when external forces are applied. Therefore, he also has sources and sinks depending on the sign of the product $\boldsymbol{X}_{k} \cdot \boldsymbol{J}_{k}$. This is consistent with both the molecular kinetic interpretation of temperature as the average velocity of disordered particle motion, and the statistical-mechanical interpretation of entropy as a measure of the amount of chaotic motion, which can decrease not only with the convective transfer of impulse across the boundaries of the system (i.e., for heat transfer) but also upon its transition into an ordered movement (impulse $\boldsymbol{P}=M \boldsymbol{v}$ ). As for locally equilibrium systems $\left(\boldsymbol{x}_{k}=0\right)$, according to (13), neither sources nor sinks of any parameters can arise in them.

Thus, a one-sided increase in entropy, i.e., its lack of flow $\sigma_{s}<0$, did not in any way follow from relation (13). This circumstance reveals the fallacy of the justification given by Clausius of the principle of increasing entropy. This can be shown in the most general and shortest way, based on the properties of the energy $U$ and the entropy $S$ as a function of the state. According to (6), any $k$-th form of energy $U_{k}=U_{k}\left(\Theta_{k}\right)$, i.e., is a function of this kind movement, characterized by the energy carrier $\Theta_{k}$ (mass $M$, entropy $S$, charge $\Theta_{e}$, impulse $\boldsymbol{P}$, etc.). Therefore, the energy of the multivariant system $U=\Sigma_{k} U_{k}$ as a function of its state has the form $U_{k}=U\left(M, S, V, \Theta_{e}, \boldsymbol{P}\right)$. In this case, considering the entropy $\mathrm{S}$ as an inverse function $S=S\left(U, M, V, \Theta_{e}, P\right)=$ const.

by virtue of the laws of conservation of energy $U$, mass $M$, volume $V$ and charge $\Theta_{e}$ and impulse $\boldsymbol{P}$ of an isolated system, it is directly convinced that its entropy $S$ also remains unchanged. Thus, remaining within the framework of equilibrium thermodynamics, it is impossible to substantiate the principle of increasing entropy [13].

It is another matter if the state variables of the system under study include non-conserved parameters, for example, the thermodynamic forces $\boldsymbol{X}_{k}$, i.e., $U=\Sigma_{k} U_{k}\left(\Theta_{k}, \boldsymbol{X}_{k}\right)$. Then the principle of increasing entropy is easily proved since it directly follows from relation (14). However, such proof will no longer be necessary, since these additional parameters $\boldsymbol{X}_{k}$ themselves are more informative, simple, and obvious criteria for evolution than entropy. A change in their absolute value directly reflects both the involution (degradation) of the systems and their evolution: $d\left|\boldsymbol{X}_{k}\right|>0$ (evolution); $d\left|\boldsymbol{X}_{k}\right|<0$ (involution), (17)

moreover, for each $k$-th degree of freedom of the multivariate system separately, which is not available entropy [13]. Thus, we are once again convinced that the reason for the inconsistency proposed by R. Clausius and his many followers of the "evidence" of the principle of increasing entropy was their attempt to take into account irreversibility, not taking into account its cause the heterogeneity of the systems under study. 


\section{Incompatibility of the principle of increasing entropy with the evolution}

The evolution of a system is traditionally understood as its development, complexity, the acquisition of new degrees of freedom, and the ability to adapt to changing environmental conditions. Therefore, the degradation of the "expanded" system, including the environment, prescribed by expression (15), really leads to a "glaring contradiction" with evolution. According to (13), any kth degree of freedom of a multivariate system can either arise in the process of evolution or disappear in the process of involution. It cannot be otherwise since the very concept of evolution assumes the presence of sources of $\sigma_{k}$ for any energy carrier $\Theta_{k}$, and their transfer from the environment is deliberately excluded by the absence of those properties in it that arise in the system due to structural changes in it.

The existence of such sources to date is excluded by the "laws" of conservation of mass, momentum, charge, which are accepted by modern physics as fundamental and having the same status as the law of conservation of energy. The justification for such statements is Noether's theorem, according to which these laws are a consequence of the homogeneity and isotropy of space and time [14]. However, this initial premise itself is of a postulating nature and, strictly speaking, refers to space, and not to the matter filling it. The density of the latter, according to modern data, ranges from $\sim 10^{-27} \mathrm{~g} \mathrm{~cm}^{-3}$ in regions of the Universe free of ordinary (baryonic) matter to $\sim 10^{18} \mathrm{~g} \mathrm{~cm}^{-3}$ in "white dwarfs" [15]. Under such conditions, there can be no talk of the homogeneity of space, since its "curvature" is determined according to GR by the presence of a mass in it. In mechanics, the law of force $\boldsymbol{F}=d \boldsymbol{P} / d t$ itself indicates the incompatibility of the laws of conservation of energy and momentum, in which the long-range forces play the role of the momentum source $\boldsymbol{P}$ (that is, that part of its change that is not related to transfer across the system boundaries). However, according to (13), they can also be the internal forces $\boldsymbol{X}_{k}$ [16].

The fact that the incompatibility of evolution with the laws of conservation of energy $\Theta_{k}$ also applies to the mass $\mathrm{M}$ of the ordinary (baryonic) matter is evidenced by recent discoveries in the field of astronomy. They led to the unequivocal conclusion that at least $95 \%$ of the mass of the Universe is "hidden" since it is not involved in thermal and electromagnetic radiation and is not observable. This is the same matter that was called ether for three centuries, and after its expulsion from physics, it was replaced by even more vague concepts of "physical vacuum", "field of matter", "dark", a matter of energy, "quintessence", etc. Reliably about it, all that is known is that it has a non-zero density $\rho_{o}$ and that it is from it that all the known forms of the ordinary (observable) substance of the Universe are formed. This means that its mass $M$ also has sources $d P / d t$, due to the condensation of the non-baryonic part of the matter of the Universe (isolation from which does not exist).

Thus, any energy carriers $\Theta_{k}$, including the entropy $S$, obey relation (13) and, following the sign of the product $\boldsymbol{X}_{k} \cdot \boldsymbol{J}_{k}$, have both sources and sinks. This means that entropy as an extensive measure of chaotic motion arises and disappears along with the baryonic form of matter in the process of its circulation in the Universe, and the question of the "thermal death" of the Universe should be considered closed.

\section{Acknowledgements:}

The author would like to thank IJoT publishing editor Dr. Ersin Sayar for his editing help.

\section{References}

1. Yastrzhembsky A. S. Thermodynamics and the history of its development. M.: Energy, 1966. (In Russian).

2. Bazarov I.P. Thermodynamics. Ed. 4th. M.: Higher School, 1991. (In Russian).

3. De Groot SR, Mazur R. Nonequilibrium thermodynamics. - Amsterdam, 1962.

4. Gyarmati I. Non-Equilibrium Thermodynamics. Field Theory and Variation Principles. Springer -Verlag, 1970

5. Landau L.D. Lifshits E.M. Theoretical physics. T.5. Statistical Physics. -M.: Science, 1964. (In Russian).

6. Klausius R. Die mechanishe Wärmetheorie. Draundschweig, Bd. I, 1876

7. Etkin V. Thermokinetics (Synthesis of Heat Engineering Theoretical Grounds). - Haifa, 2010; Etkin V.A. Thermokinetics (Thermodynamics of nonequilibrium processes of energy transfer and conversion). Tolyatti, 1999. (In Russian).

8. Umov A. I. Selected Works. M.L., 1950. (In Russian). 
9. Haase R. Thermodynamik der irreversiblen processe. Darmstadt, 1963.

10. Etkin V.A. Heat and work in irreversible processes // Izv. universities. Energy, 4 (1988). 118 -122. (In Russian).

11. Onsager L. Reciprocal relations in irreversible processes. // Phys. Rev. 1931. -- 237 (14). P.405-426; 238 (12). - P.2265-2279.

12. Prigogine I. Time, structure and fluctuations (Nobel lecture on chemistry in 1977) //Uspekhi fiz. nauk. 131(1980).185-207. (In Russian).

13. Etkin V.A. New Criteria of Evolution and Involution of the Isolated Systems. // International Journal of Thermodynamics (IJoT) 2018, 21 (2), pp. 120-126, doi: 10.5541 / ijot.341037

14. Noether E. Invariant variational problems // Variational principles of mechanics / Ed. Polak L.S. M., Fizmatlit, 1959.613-614. (In Russian).

15. Bahcall, Neta A. Large-scale structure in the universe indicated by galaxy clusters. // Annual review of astronomy and astrophysics. 26 (1988). 631-686.

16. Etkin V. On the incompatibility of the laws of conservation of energy and momentum. // Annali d'Italia, 3 (2020).41-47 (In Russian). 